\title{
Disks formed by Rotation Induced Bi-stability
}

\author{
Henny J.G.L.M. Lamers ${ }^{1,2}$, Jorick S. Vink ${ }^{1}$, Alex de Koter ${ }^{3}$,
} and Joseph P. Cassinelli ${ }^{4}$

1 Astronomical Institute, Utrecht University, Princetonplein 5, NL-3584 CC, Utrecht, The Netherlands

${ }^{2}$ SRON Laboratory for Space Research, Utrecht, The Netherlands

3 Astronomical Institute, University of Amsterdam, Kruislaan 403, NL-1098 SJ, Amsterdam, The Netherlands

${ }^{4}$ Dept of Astronomy, University of Wisconsin, $475 \mathrm{~N}$ Charterstreet, Madison, WI 53706-1582, Madison, USA

\begin{abstract}
We discuss the evidence for the existence of bi-stable stellar winds of early type stars, both theoretically and observationally. The ratio between the terminal wind velocity and the escape velocity drops steeply from about 2.6 for stars with $T_{\text {eff }}>21000 \mathrm{~K}$ to about 1.3 at $T_{\text {eff }}<21000 \mathrm{~K}$. This is the bi-stability jump, which is due to a change in the ionization of the wind and in the wind driving lines. The mass loss rate increases across the jump by about a factor 2 to 5 from the hotter to the cooler stars. The mass flux from rapidly rotating stars can also show the bi-stability jump at some lattitude between the pole and the equator, with a slow high density wind in the equatorial region and a faster low density wind from the poles. This might explain the disks of rapidly rotating $\mathrm{B}[\mathrm{e}] \mathrm{stars}$, formed by the Rotation Induced Bi-stability mechanism. We discuss the RIB mechanism and its properties. We also describe some future improvements of the model.
\end{abstract}

\section{Introduction}

The concept of bi-stability of the winds of early type stars was first described by Pauldrach and Puls (1990, hereafter P\&P) based on their calculations of the line driven wind of $\mathrm{P}$ Cygni. They showed that the wind of a star with $T_{\text {eff }}=19300 \mathrm{~K}$ can be either dense and slow if the wind is driven by lines in the Balmer continuum, or fast and less dense if the driving is due to lines in the Lyman continuum. The change occurs over a small range of $T_{\text {eff }}$ or gravity because the optical depth in the Lyman continuum is very sensitive to $T$ and $\rho$ in the wind near $T_{\text {eff }} \simeq 20000 \mathrm{~K}$. The change from the fast to the slow wind solution is called the bi-stability jump. Lamers et al. (1995) found in a study of the terminal velocities of stellar winds that the bi-stability jump also occurs for normal OB supergiants near about type B1, where the ratio $v_{\infty} / v_{\text {esc }}$ drops steeply from about 2.6 for the hotter stars to 1.3 for the cooler stars. Lamers and Pauldrach (1991, hereafter L\&P) showed that the same bi-stability may occur in the wind of a rapidly rotating early-B star between the hotter pole and the cooler equator. This will lead to a fast low density wind from the pole and a slow dense wind from the equator, i.e. a disk like structure. This mechanism is called the Rotation Induced Bi-stability or RIB. 


\section{The bi-stability of the winds of normal stars.}

Fig. 1a shows the ratio $v_{\infty} / v_{\text {esc }}$ from Lamers et al. (1995) as a function of $T_{\text {eff }}$ for normal OB supergiants. There is a clear jump near $T_{\text {eff }} \simeq 21000$ or spectral type B1. (A similar jump in $v_{\infty} / v_{\text {esc }}$ may be present near $10000 \mathrm{~K}$ ). Fig. $1 \mathrm{~b}$ shows the same data but now in terms of $v_{\infty}$ versus $v_{\text {esc }}$ in different

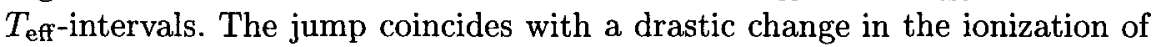
the wind: stars on the "hot side" of the jump have strong CIV and weak CII wind lines, and the situation is reversed for stars on the "cool side" of the jump (see Fig. 8 of Lamers et al. 1995).
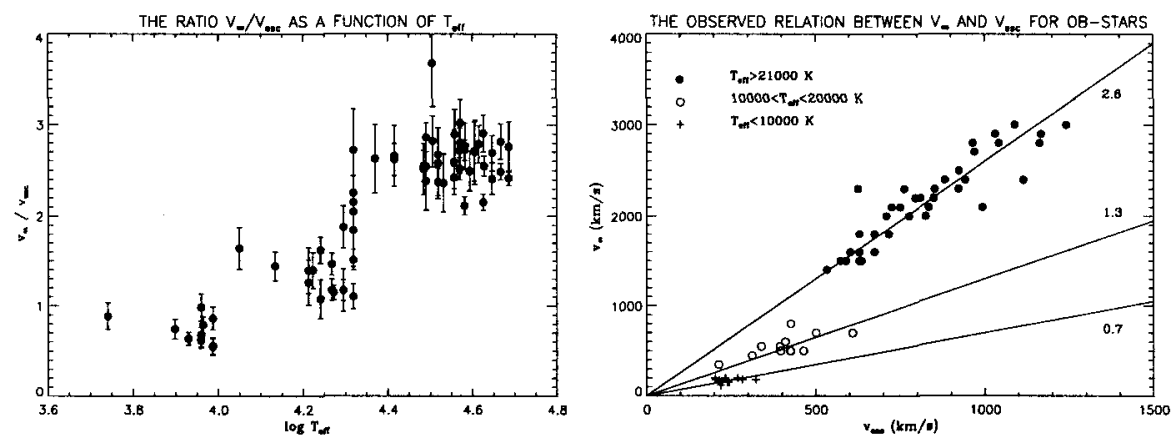

Fig. 1. Left: the observed ratio $v_{\infty} / v_{\text {esc }}$ for supergiants as a function of $T_{\text {eff }}$. Notice the jump near $T_{\text {eff }} \simeq 21000 \mathrm{~K}$, and possibly also near $T_{\text {eff }} \simeq 10000 \mathrm{~K}$. Right: the same data plotted as $v_{\infty}$ versus $v_{\text {esc }}$ (from Lamers et al., 1995).

The bi-stability model predicts that the mass loss rate should also show a jump, with $\dot{M}$ increasing by about a factor 2 to 5 when $v_{\infty}$ decreases (P\&P and L\&P). The counteracting behaviour of $\dot{M}$ and $v_{\infty}$ indicate that the wind momentum loss $\dot{M} v_{\infty}$ is approximately invariant across the bi-stability jump. This may be expected as the ratio between the radiative momentum loss, $L / c$, and the wind momentum loss hardly changes across the jump. It should be said that observational support for this statement is presently meagre, mainly because of the difficulty in deriving mass loss rates from UV lines with uncertain degrees of ionization.

Vink et al. (1999) have calculated the mass loss rates as a function of

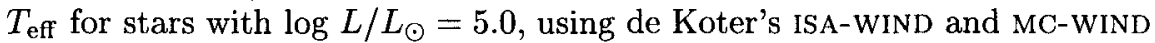
codes (de Koter et al. 1993 and 1997). The first program solves the statistical equilibrium and radiative transfer equations for extended atmospheres and winds, with $\mathrm{H}, \mathrm{He}, \mathrm{C}, \mathrm{N}, \mathrm{O}$ and $\mathrm{Si}$ in non-LTE. The mass loss rate $\dot{M}$, and the velocity law, specified by $\beta$ and $v_{\infty}$, are input parameters. The second program calculates the total radiation pressure due to the continuum and lines, including about $10^{5}$ of the strongest lines, mainly of the iron group 
elements. The mass loss rate is found by requiring that the radiative momentum input into the wind is equal to the wind momentum (see Abbott \& Lucy, 1985 and Lucy \& Abbott, 1993 for details). Fig. 2a shows the mass loss rates as a function of $T_{\text {eff }}$ for wind models with values of $v_{\infty} / v_{\text {esc }}=2.6,2.0$ and 1.3 respectively. From the observations we know that the ratio $v_{\infty} / v_{\text {esc }}$ decreases steeply from 2.6 to 1.3 near $21000 \mathrm{~K}$. Adopting these ratios we find the variation in mass loss rate as shown in Fig. $2 \mathrm{~b}$. This figure shows a steep increase in $\dot{M}$ of about a factor five between $T_{\text {eff }}=26000$ and $20000 \mathrm{~K}$. This is due to a change in the driving lines in this temperature range (Vink et al. 1999).
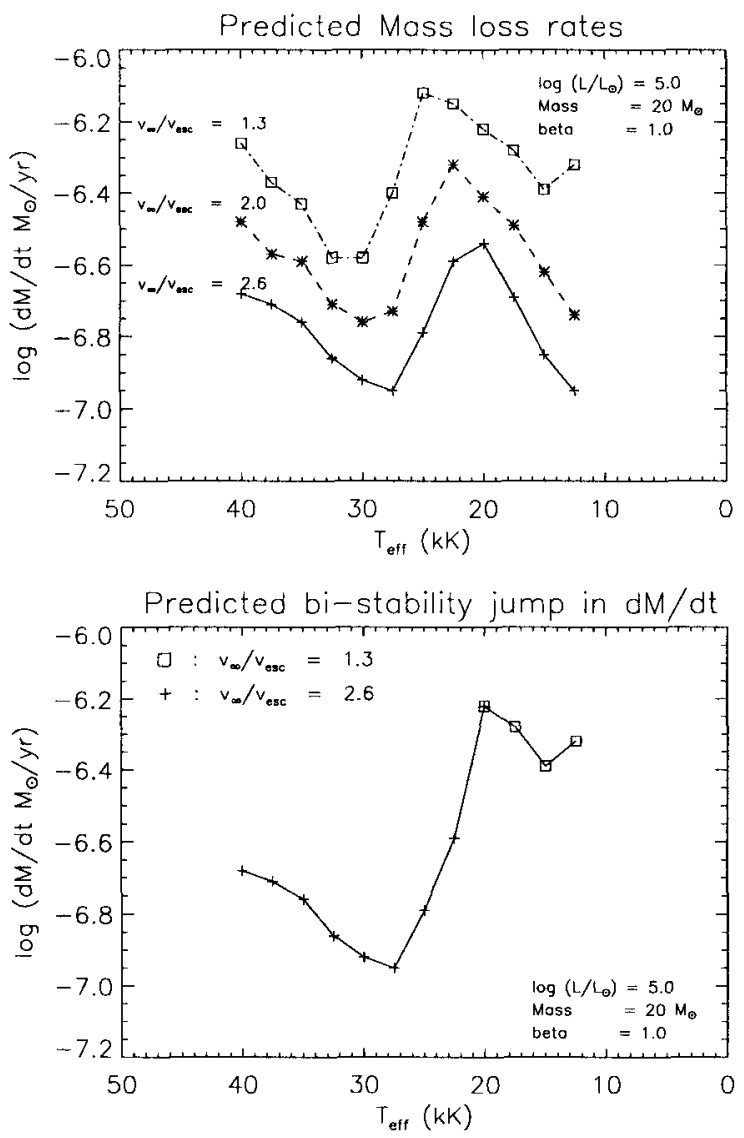

Fig. 2. Top: the predicted mass loss rates as function of $T_{\text {eff }}$ for a grid of models with three values of $v_{\infty} / v_{\text {esc }}$. Bottom: the mass loss rate as a function of $T_{\text {eff }}$, for winds with the observed ratio $v_{\infty} / v_{\text {esc }}=2.6$ if $T_{\text {eff }}>21000$ and 1.3 if $T_{\text {eff }}<21000$ K (from Vink et al., 1999). 


\section{Rotation-Induced Bi-stability}

$\mathrm{P} \& \mathrm{P}$ and $\mathrm{L} \& \mathrm{P}$ showed that line driven winds from early-B stars can change from low density and high velocity to high density and low velocity if the optical depth of the wind in the Lyman continuum becomes larger than about unity. The basic idea of the RIB model is that the same transition can occur in the wind of a rotating early B star between the pole and the equator. This is because the reduction of the effective gravity and $T_{\text {eff }}$ between the pole and the equator due to rotation enhances the optical depth in the wind from the pole to the equator. If the optical depth changes from a value smaller than unity at the pole, to a value larger than unity at the equator, the bi-stability jump will occur at some intermediate latitude. If this happens, the equatorial wind will be slow and dense, whereas the polar wind will be fast and tenuous. This is very similar to the observed characteristics of B stars with outflowing disks. An attractive feature of the RIB mechanism is that it is expected to work only for early B stars, because the models and the observations show that the bi-stability can occur around $T_{\text {eff }}$ about $20000 \mathrm{~K}$. (The exact value of $T_{\text {eff }}$, where the bi-stability jump occurs, depends on luminosity, gravity and rotational velocity.)

Figure (3) illustrates the nature of the wind predicted by the RIB model for $\mathrm{B}[\mathrm{e}]$ supergiants. This picture is quite similar to the empirical model of these stars derived by Zickgraf et al. (1986).

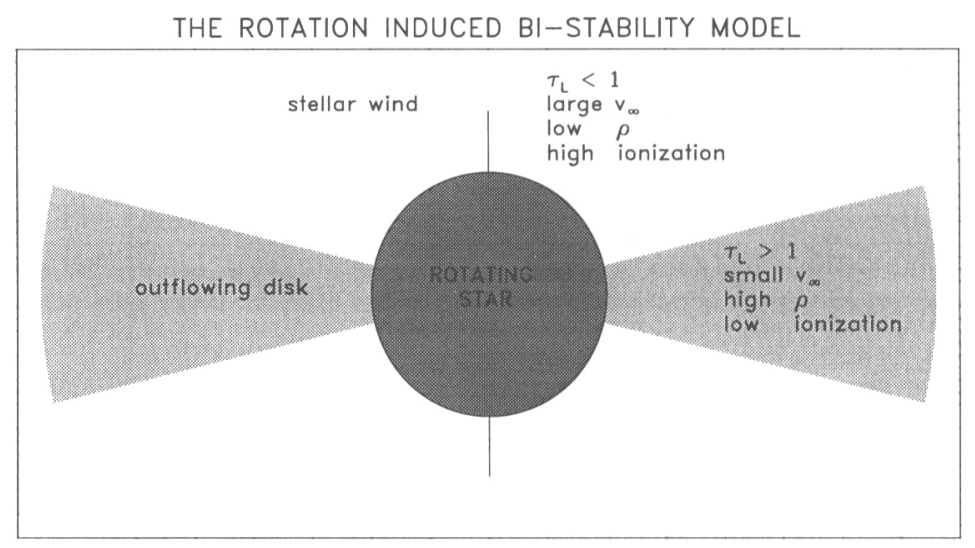

Fig. 3. A schematic picture of a rapidly rotating $B[e]$ supergiant formed by the bi-stability mechanism. The wind is optically thin, $\left(\tau_{L}<1\right)$ in the polar regions, and so the wind there has a high velocity, high ionization state and low density. The wind is thick $\left(\tau_{L}>3\right)$ in the equatorial region so the wind there has a low velocity, lower ionization, and a higher density. (From Lamers and Pauldrach, 1991) 
Since the bi-stability depends on the optical depth of the wind in the Lyman continuum, we consider the various effects of stellar rotation on the wind parameters and optical depth, following L\&P.

The net surface gravity, $g_{\text {net }}$, is given as a function of the polar angle $\theta$ on the star by

$$
g_{\text {net }}(\theta)=\frac{G M_{*}\left(1-\Gamma_{e}\right)}{R(\theta)^{2}}\left(1-\omega^{2} \sin ^{2} \theta\right)
$$

with $\theta=0$ and $\pi$ at the poles and $\pi / 2$ at the equator and with $\omega<1$ defined by

$$
\omega \equiv \frac{v_{\mathrm{rot}}}{v_{\mathrm{crit}}}=v_{\mathrm{rot}} \sqrt{\frac{R_{*}}{G M_{*}\left(1-\Gamma_{e}\right)}}=\Omega \sqrt{\frac{R_{*}{ }^{3}}{G M_{*}\left(1-\Gamma_{e}\right)}}
$$

We ignore the rotational distortion of the star, so $R(\theta)=R_{*}$. According to the von Zeipel theorem the radiative flux at the stellar surface is proportional to the local gravity, so

$$
T_{\text {eff }}^{4}(\theta) \sim g_{\text {net }}(\theta) \sim\left(1-\omega^{2} \sin ^{2} \theta\right)
$$

The changes in gravity and radiation temperature as a function of $\theta$ affect the wind in several ways.

(a) The wind speed in the radiation driven wind theory is proportional to the escape speed. Consequently, the wind speed at $\theta$ is

$$
v_{\infty}(\theta) \sim v_{\text {esc }}(\theta) \sim \sqrt{R_{*} g_{\text {net }}(\theta)} \sim\left(1-\omega^{2} \sin ^{2} \theta\right)^{0.5}
$$

So the wind speed decreases from the pole to the equator.

(b) In the piecewise spherical model that we consider here, the mass flux of a radiation driven wind, $F_{m}$, depends on the local radiative flux, i.e. on $T_{\text {eff }}(\theta)^{4}$, and on the local effective gravity $g_{\text {eff }}$. From the mass loss calculations of Vink et al. (1999) for $\log L / L_{\odot}=5.0$ and for different masses and effective temperatures we find that in the range of $20000 \leq T_{\text {eff }} \leq 30000 \mathrm{~K}$ the mass flux can be written as

$$
F_{m} \sim\left\{T_{\mathrm{eff}}{ }^{4}(\theta)\right\}^{1.66} g_{\text {net }}^{-1.2} \sim\left(1-\omega^{2} \sin ^{2} \theta\right)^{+0.5}
$$

This means that the mass flux decreases slightly from the pole to the equator.

(c) The Lyman continuum flux is very sensitive to the $T_{\text {eff }}$. The brightness temperature in the Lyman continuum scales as $T_{L} \sim T_{\text {eff }}{ }^{1.6}$ for early-B stars (Kurucz, 1979). The fraction of neutral $\mathrm{H}$ is inversely proportional to the photo-ionization rate $R_{i}=4 \pi \int_{\nu_{0}}^{\infty}\left(J_{\nu} / h \nu\right) a_{\nu} d \nu$, which varies as $R_{i} \sim T_{L}^{9.7}$ in the range of $15000<T_{L}<20000 \mathrm{~K}$, corresponding to about $20000<$ $T_{\text {eff }}<25000 \mathrm{~K}$. So we find that the ionization rate of neutral $\mathrm{H}$ is

$$
R_{i} \sim T_{\mathrm{eff}}^{15.5} \sim\left(1-\omega^{2} \sin ^{2} \theta\right)^{3.9}
$$

(d) The optical depth in the Lyman continuum through the wind, $\tau_{L}$, is proportional to the column density of neutral $\mathrm{H}$. As this is determined by ionization and recombination, $\tau_{L}$ is proportional to $\rho^{2} \alpha / R_{i}$, where $\alpha \sim T^{-0.5}$ 
is the recombination rate. Accounting for the dependence of the optical depth on both the ionization and recombination rate and on the column density, we find that the optical depth of the wind in the Lyman continuum varies with polar angle approximately as

$$
\tau_{L} \sim\left\{\frac{F_{m}(\theta)}{v_{\infty}(\theta)}\right\}^{2} \frac{\alpha}{R_{i}} \sim\left\{\frac{F_{m}(\theta)}{v_{\infty}(\theta)}\right\}^{2} T_{\text {eff }}{ }^{-16.0} \sim\left(1-\omega^{2} \sin ^{2} \theta\right)^{-4.1}
$$

This relation implies that an early $\mathrm{B}$ star that is rotating will have a larger wind optical depth in the Lyman continuum in the equatorial region than near the polar region. The difference will be a factor of 16 for a star that is rotating with $\omega=0.7$ and a factor of 3 if $\omega=0.5$.

In this estimate we have assumed that the velocity law $v(r) / v_{\infty}$ is constant for all polar angles. If the velocity law is slower (larger $\beta$ ) for lower temperatures, as suggested by the study of velocity laws of B-stars (Rivinius et al. 1997), the optical depth will depend even stronger on $\theta$ than given by eq. (7).

P\&P and L\&P have shown that the bi-stability jump occurs when the optical depth of the wind in the Lyman continuum at a wavelength of $600 \AA$ reaches a value of $\tau_{L} \simeq 1$ (see Fig. 1 of $L \& P$ ), where $\tau_{L}$ is the optical depth of the wind from infinity to the sonic point.

This implies that the half opening angle is given by the condition

$$
\left(1-\omega^{2} \sin ^{2} \theta\right)^{-4.1} \simeq 1 / \tau_{L} \text { (pole) }
$$

or

$$
\theta \simeq \arcsin \left\{\frac{1}{\omega}\left[1-\tau_{L}(\text { pole })^{1 / 4.1}\right]^{0.5}\right\}
$$

Fig. 4 shows the half opening angle of the RIB-disk of a B-type supergiant of $T_{\text {eff }}=20000 \mathrm{~K}, M_{*}=35 M_{\odot}, L=5 \times 10^{5} L_{\odot}$ as a function of $\omega$ for different polar mass loss rates defined by $\dot{M}_{\text {pole }}=4 \pi R_{*}^{2} F_{m}$ (pole). The polar optical depth of the wind in the Lyman continuum at $600 \AA$ is about 0.05 if $\dot{M}_{\text {pole }}=$ $2 \times 10^{-6} M_{\odot} \mathrm{yr}^{-1}$. This is a typical value for the polar mass loss rate of $\mathrm{B}[\mathrm{e}]$ stars (Zickgraf et al., 1986).

\section{Conclusions and future work}

The RIB model uses the properties of rotating stars such as the von Zeipel theorem to show that the bi-stability jump of a rotating star may occur between the pole and the equator. This will lead to a high density and low velocity wind from the equator and a low density and high velocity wind from the pole. The density contrast is about a factor of ten. This contrast is similar to what is observed for the $\mathrm{B}[\mathrm{e}]$ supergiants. So the RIB mechanism might explain the winds and disks of $\mathrm{B}[\mathrm{e}]$ stars if the stars are rapidly rotating. An 


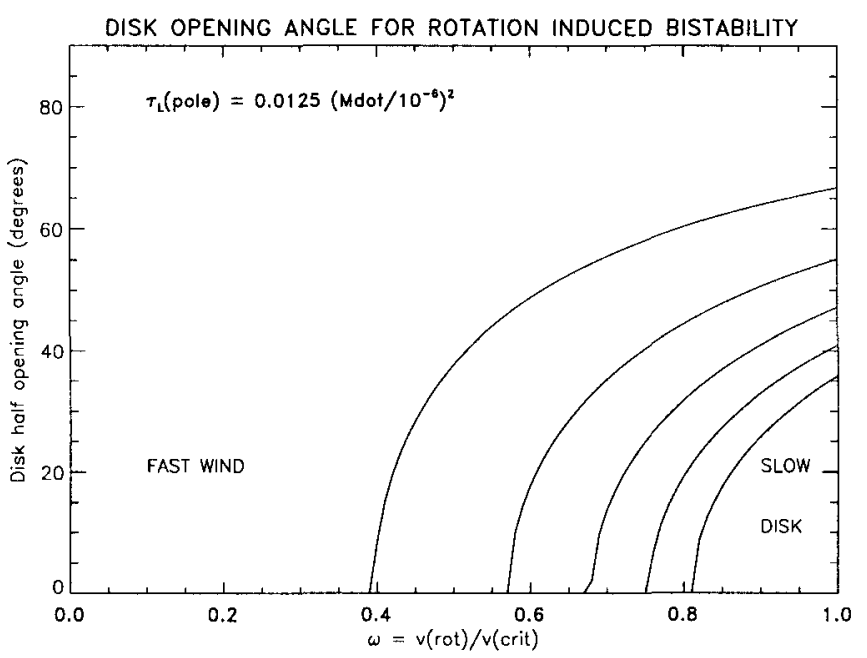

Fig. 4. The half opening angle of the disk of a star with $T_{\text {eff }}=20000 \mathrm{~K}, M=35 M_{\odot}$, $L=5 \times 10^{5} L_{\odot}$ as a function of $\omega$ for different polar mass loss rates: $\log \left(\dot{M}_{\text {pole }}\right)=$ -5.2 (upper curve), $-5.4,-5.6,-5.8$ and -6.0 (lower curve) respectively. The observed polar mass loss rate of $\mathrm{B}[\mathrm{e}]$ stars is $\log \left(\dot{M}_{\text {pole }}\right) \simeq-5.7$.

attractive feature of the RIB mechanism is that it is expected to work only for the early-B stars, which agrees with the spectral types of the B[e] stars!

The mechanism described by L\&P and in this paper is very descriptive and simplified. The major simplification is the piecewise spherical concept, where we use the information about the dependence of the mass flux on the local quantities $T_{\text {eff }}(\theta)$ and $g_{\text {eff }}(\theta)$ derived from the observations and theory of winds from normal, slowly rotating stars. We are presently studying the formation of disks by the RIB mechanism in a better approximation where the radiation pressure is calculated from the proper distribution of the radiation over the surface of the star (Pelupessy and Lamers, in preparation).

The disks produced by the RIB mechanism are rather wide, with full opening angles on the order of 90 degrees or more. Possibly the wind compression effect (Bjorkman and Cassinelli, 1993 and Bjorkman, these proceedings) will compress the disks to smaller opening angles. The differences between the RIB and the Wind Compressed Disk model (WCD) are:

(a) In the RIB model the disk is due to the higher equatorial mass flux, whereas in the WCD model the disk is due to the compression of the wind towards the equator.

(b) The RIB model can give at most a factor of ten density contrast from equator to pole and works for $\mathrm{B}$ supergiants with high mass loss rates. The WCD model can produce a higher density contrast, by an orbital effect that is independent of mass loss rate. 
The combination of the bi-stability mechanism and the WCD mechanism will result in even higher density contrasts: the bi-stability producing a higher mass flux from the equator, and the WCD effect concentrating the flow towards the equator.

The formation of disks by the RIB mechanism depends on the stellar rotation. Maheswaran and Cassinelli (1994) have calculated the rotation history of massive stars as a function of mass loss rate for different mass loss mechanisms. They have shown that if there is no significant increase in angular velocity inward from the surface during the main sequence and the early supergiant phase, then the equatorial rotation rate will be extremely small before the star reaches the $\mathrm{B}[\mathrm{e}]$ phase and the star will be an almost nonrotator during all subsequent evolution phases. The existence of $B[e]$ stars shows that this is not the case and the stars survive the main sequence phase and the early supergiant phases with sufficient angular momentum in the envelope. Hegel and Langer (see Langer, these proceedings) have shown that massive stars returning from the red supergiant phase by contraction will rotate almost rigidly, because of the short convection turn-over time. This will result in a speeding up of the star when it moves to the left. So we expect that a massive star can have a $\mathrm{B}[\mathrm{e}]$ disk in two phases: the most rapidly rotating stars may immediately become B[e] stars when they evolve off the main sequence. Mildly rotating stars can devellop a $\mathrm{B}[\mathrm{e}]$ disk when they return from the RSG phase with a significantly smaller mass and angular momentum than during the first crossing.

\section{References}

Abbott, D.C., Lucy, L.B. 1985, Ap.J 288, 679

Bjorkman, J.E., Cassinelli, J.P. 1993, Ap.J 409, 429

de Koter, A., Schmutz, W., Lamers, H.J.G.L.M. 1993, A\&A 277, 561

de Koter, A. Heap, S.R., Hubeny, I. 1997, Ap.J. 477, 792

Kurucz, R.L. 1979, Ap.J.Supl 40, 1

Lamers, H.J.G.L.M., Pauldrach, A.W.A. 1991, $A \& A$ 244, L5 (L\&P)

Lamers, H.J.G.L.M., Snow, T.P, Lindholm, D.M. 1995, Ap.J. 455, 269

Langer, N. 1999 (these proceedings)

Lucy, L.B., Abbott, D.C. 1993 Ap.J. 405, 738

Mahareswaran, M., Cassinelli, J. 1994, Ap.J. 421, 718

Pauldrach, A.W.A., Puls, J. 1990, $A \& A$ 237, 409 (P\&P)

Pelupessy, I., Lamers, H.J.G.L.M. 1999 (in preparation)

Rivinius, Th. et al. 1997, $A \& A$ 318, 819

Vink, J.S., de Koter, A., Lamers, H.J.G.L.M. 1999 (in preparation)

Zickgraf, F.J. et al. 1986, $A \mathscr{G} A, \mathbf{1 6 3}, 119$

Zickgraf, F.J. 1999 (these proceedings) 


\section{Discussion}

M. Friedjung: What is the wind velocity? Taking S 22, which I studied some years ago, you see narrow, optically thick Fe II lines in the optical without $\mathrm{P}$ Cygni components. On the other hand, the Balmer lines have P Cygni components at about $100 \mathrm{~km} / \mathrm{s}$. This seems also to be the case for the optically much thicker UV Fe sc ii lines. If the disk is edge on, you must have a very slow non-classical wind in the plane of the disk.

R. Ignace: Using the von Zeipel theorem, Owocki et al. indicated that $\dot{M} \sim$ $g_{\text {eff }}$, in contrast to $g_{\text {eff }}^{-1.5}$ that you use. This will make $\tau_{\mathrm{L}}(\Theta)$ a less-sensitive function of latitude, and the disk opening angle may not be such a strong function of the rotation.

S. Owocki: I agree. Henny seems to be overlooking the flux dependence of the mass-loss rate variation. This will work against the equatorial increase in density for the bi-stability jump.

A. Maeder: Your bi-stability model predicts that there should be a discontinuity in the mass-loss rates (for slowly rotating stars) around $20000 \mathrm{~K}$. Do we observe it?

H. Lamers: This requires a mass-loss study of early B stars based on $P$ Cygni profiles. It has not been done yet, probably because we do not know the ionisation structure of the winds of B supergiants.

E. Verdugo: What does the jump between the B supergiants and the A supergiants mean? Is it real?

H. Lamers: The figure showing $v_{\infty}$ versus $v_{\text {eff }}$ indeed suggests a second bistability jump near $10000 \mathrm{~K}$. However, this depends on the observations of only a few stars, with $\mathrm{T}_{\text {eff }} \sim 10000 \mathrm{~K}$. So at the moment we can say that $v_{\infty} / v_{\text {esc }}$ decreases as $\mathrm{T}_{\text {eff }}$ decreases between $\mathrm{B}$ and $\mathrm{A}$ stars, but whether it does so discontinuously remains to be confirmed.

G. Koenigsberger: If a star's wind is exactly at the bi-stability limit, can it flip from one state to another?

H. Lamers: Yes. This was proposed for P Cygni by Pauldrach and Puls (1989). They suggest the following scenario: if the star is on the cool side of the bi-stability limit, the high mass loss rate will result in strong wind blanketing. This will heat up the photosphere and move the star to the hot side of the bi-stability limit. The decreased mass-loss rate will then reduce the blanketing, the atmosphere will cool and the star will cross the bi-stability jump again. They estimate the fluctuation time, i.e., the time to build up and destroy the blanketing layers, to be of the order of one month or so for P Cygni.

K. Gayley: In the absence of bi-stability, the gravity darkening would increase the mass loss through the polar region. Are you saying that in spite 
of that, bi-stability will reverse that effect? Will mass loss then peak at an intermediate angle where the transition occurs?

H. Lamers: There are two opposing effect: (a) the increase of $\mathrm{T}_{\text {eff }}$ to the pole, which implies a higher flux $\left(\mathrm{F}_{\mathrm{rad}} \sim \mathrm{T}^{4}\right)$; and (b) the increase of $g_{\text {eff }}$ towards the pole. The first effect results in an increase of the mass flux; the second in a decrease to the pole. If $\mathrm{F}_{\mathrm{m}} \sim g_{\text {net }} M-3-1.5 \mathrm{~F}_{\mathrm{rad}}^{+1.5}$ (as suggested by the models) then the two effects cancel because of the von Zeipel effect: $\mathrm{F}_{\mathrm{rad}} \sim \mathrm{T}^{4} \sim g$. Subtle effects may affect the $\dot{\mathrm{M}}(\Theta)$ dependance either way.

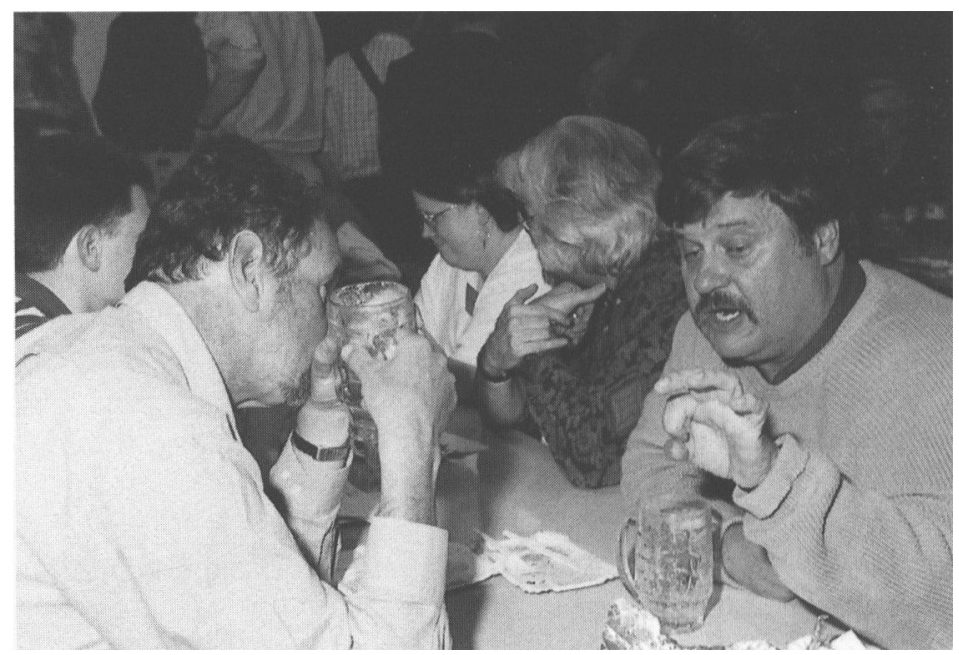

Henny Lamers and Stan Owocki 